\title{
Assessing the impact of TRAP laws on abortion and women's health in the USA: a systematic review
}

\author{
Nichole Austin, Sam Harper
}

\begin{abstract}
- Additional material is published online only. To view please visit the journal online (http://dx.doi.org/10.1136/ bmjsrh-2017-101866).
\end{abstract}

Department of Epidemiology, Biostatistics and Occupational Health, McGill University, Montreal, Canada

\section{Correspondence to}

Nichole Austin, Department of Epidemiology, Biostatistics and Occupational Health, McGill University, Montreal H3A 1A2, Canada; nichole.austin@mail. mcgill.ca

Received 12 July 2017 Revised 26 January 2018 Accepted 6 February 2018 Published Online First 9 March 2018

\section{SLinked}

- http://dx.doi.org/10.1136/ bmjsrh-2017-200042

Check for updates

To cite: Austin N, Harper S. BMJ Sex Reprod Health 2018:44:128-134.

\begin{abstract}
Introduction Targeted Regulation of Abortion Providers (TRAP) laws impose extensive and sometimes costly requirements on abortion providers and facilities, potentially leading to barriers to care. Understanding the impact of these laws is important given their prevalence in the USA, but no review to date has summarised the available evidence. We conducted a systematic review of literature on TRAP laws and their impact on abortion trends and women's health.

Methods We searched MEDLINE, PubMed and EconLit for original, quantitative studies where the exposure was at least one TRAP policy and

\section{Key messages}

- Targeted Regulation of Abortion Providers (TRAP) laws are common in the USA, but there is limited quantitative evidence on their effects on women's health.

- Certain TRAP laws may be associated with a decrease in state-level abortion rates.

- Additional research is required to better understand the impact of these laws on women's health outcomes.
\end{abstract} the outcome was abortion and/or any women's physical or mental health outcome.

Results Six articles met our inclusion criteria. The most common outcome was population-level abortion trends; studies also assessed the effect of TRAP laws on gestational age at presentation and measures of self-perceived burden. While certain TRAP laws (eg, admitting privilege requirements) appeared to have an effect on abortion outcomes, the impact of other laws - or combinations of laws - was unclear, due in part to heterogeneity between studies with respect to study design, geography, and exposure definition.

Conclusions TRAP laws may have an impact on the experience of obtaining an abortion in the USA.

However, our review revealed a paucity of empirical research on their population and individual-level impact, as well as some disagreement about the effect of different TRAP laws on subsequent abortion outcomes. Future research should prioritise the specific TRAP laws that may have a uniquely strong effect on state-level abortion rates and other outcomes.

\section{INTRODUCTION}

Abortion access in the USA is driven in large part by state-level regulations, yielding considerable regional variation in access to care. States rely on a range of different strategies to regulate abortion access: some policies, like mandatory ultrasound requirements and pre-abortion waiting periods, aim to regulate the individual-level demand for abortion (these are often referred to as 'demand-side' policies). There are also 'supply-side' policies, which instead regulate providers and the general provision of abortion by instituting facility/licensing requirements and gestational age limits on abortion. ${ }^{1}$ Both types of restriction are exceedingly common in the USA: over half of all states had four or more restrictions in place as of $2016,{ }^{2}$ yielding important state-

Targeted Regulation of Abortion Providers (TRAP) laws are a key type of supply-side policy and a relatively recent legislative phenomenon. ${ }^{3}$ The individual laws beneath the 'TRAP' umbrella are diverse, with regulations ranging from minor to potentially insurmountable. Common TRAP laws require abortion clinics to be located within a specified radius of a hospital; require clinics to convert to, and function as, ambulatory surgical centres (ASCs); and require providers to secure admitting privileges at a nearby hospital. Advocates of these laws argue that they play an important role in protecting women's health, but it is widely accepted that regulated abortion procedures are already exceedingly safe. ${ }^{4}$ Existing level differences in access to abortion. 
evidence suggests that certain TRAP laws pose important challenges to abortion providers and facilities, ${ }^{5}$ which is unsurprising since many laws involve costly and/or logistically challenging modifications to facilities and staff. In particular, laws requiring clinics to convert their facilities to ASCs and requiring providers to hold admitting privileges at nearby hospitals appear to decrease provider availability. ${ }^{3}$ Evidence of the impact of TRAP laws on clinic or service availability, coupled with the lack of medical necessity for these laws, prompted the American College of Obstetricians and Gynecologists in 2014 to call for an end to these policies. ${ }^{6}$

If TRAP laws do reduce provider availability or close clinics, they may also be associated with a change in US abortion rates, which have been steadily declining over the past two decades. ${ }^{7}$ Although TRAP laws probably do not explain the entire decline in abortion, the loss of even a single provider may have a profound effect on local service availability, ${ }^{17}$ and increased distance to abortion providers is associated with a decrease in abortion rates. ${ }^{58}$ However, TRAP laws may not need to close clinics to have an impact on health and other outcomes: some laws may instead increase service costs or decrease availability of appointment slots, both of which could increase the time it takes for a woman to obtain an abortion. An increase in gestational age at presentation may limit the number of providers willing to perform an abortion (particularly if the pregnancy has entered the second trimester) and increase out-of-pocket costs to patients. ${ }^{9}$ While women with adequate resources are generally able to obtain an abortion with minimal difficulty, regardless of local policies, access-oriented barriers to abortion may introduce special challenges to low-income, young and/ or rural women, as these women may be less able to manage increases in cost and distance. ${ }^{10}$

Empirical evidence on the impact of TRAP laws on abortion trends and other health outcomes has not yet been summarised in a systematic way. Literature reviews exist on demand-side policies such as parental notification laws and mandatory waiting periods, ${ }^{11-13}$ but no review to date has assessed the totality of existing literature on supply-side/TRAP laws. Given the ubiquity of TRAP laws, as well as the recent legislation against them ${ }^{14}$ it is important to understand their impact. We therefore sought to bridge this gap and summarise what is currently known about the impact of TRAP laws on abortion rates and women's health outcomes.

\section{METHODS}

We registered this review in Prospero (ID\# CRD42016039553), an international database of systematic reviews in the health and social sciences. The review consisted of four phases: title screening, abstract screening, full-text screening, and data abstraction (stage-specific inclusion/exclusion criteria are described in online supplementary appendix 1). Each phase was completed independently by two reviewers (NA/JM or NA/FU); disagreements were resolved by a third party $(\mathrm{SH})$. We conducted a systematic search for literature on TRAP/ supply-side policies and health outcomes using three electronic databases (MEDLINE, PubMed and EconLit); we also scanned the reference lists of relevant articles for additional publications. In the interest of capturing as much pertinent literature as possible, particularly since TRAP laws are sometimes assessed in conjunction with other abortion policies, we allowed our search strings to be fairly broad; our PubMed string, for example, included the following $\mathrm{MeSH}$ terms and keywords:

(Abortion, Induced OR Abortion, Legal/legislation \& jurisprudence OR Abortion, Induced/statistics \& numerical data OR Abortion, Induced/trends)

AND

(('abortion rate' OR 'abortion rates')

OR

(health OR disease* OR morbidity OR mental health OR depression)) (AND United States))

We retained quantitative research articles focused on the USA where the exposure of interest was at least one TRAP policy, and the outcome was population and/or individual-level (women's) health outcomes. We did not impose any language restrictions on our search. For the purposes of our review, specific TRAP policies included: ambulatory surgical centre (ASC) conversion requirements, other facility requirements (ie, room/corridor size specification), distance to hospital, transfer agreements, and admitting privileges at a nearby hospital (or comparable agreement), in accordance with the classification scheme currently employed by the Guttmacher Institute (https:// www.guttmacher.org/state-policy/explore/targetedregulation-abortion-providers). When articles discussed several policies, we focused on specific TRAP policies and their impact on relevant outcomes. We excluded (at the full-text phase) articles discussing changes in provider availability without a formal discussion of the TRAP policies that may have led to these changes.

At the data extraction stage, reviewers used piloted data collection forms to capture the primary policy (or policies) of interest and primary outcome measure(s), in addition to supplementary information on study design, sampling/data sources, analytical methods, effect estimates, and a subjective assessment of study quality (including reviewers' specific concerns about opportunities for bias). We used the Newcastle-Ottawa scale (NOS) as a secondary assessment of study quality. ${ }^{15}$ The NOS score ranges from 0 to 9 , and most work to date considers a score of 7 or greater evidence of 'high quality'. ${ }^{16}$ While this scale offers a basic sense of study quality, we relied primarily on reviewers' specific concerns to gauge opportunities for bias, as we found this to be a more comprehensive review strategy. This search began in June 2016; findings are current as of January 2017.

\section{RESULTS}

Our search returned 2563 unique articles, of which 329 were screened at the abstract stage and 69 were 


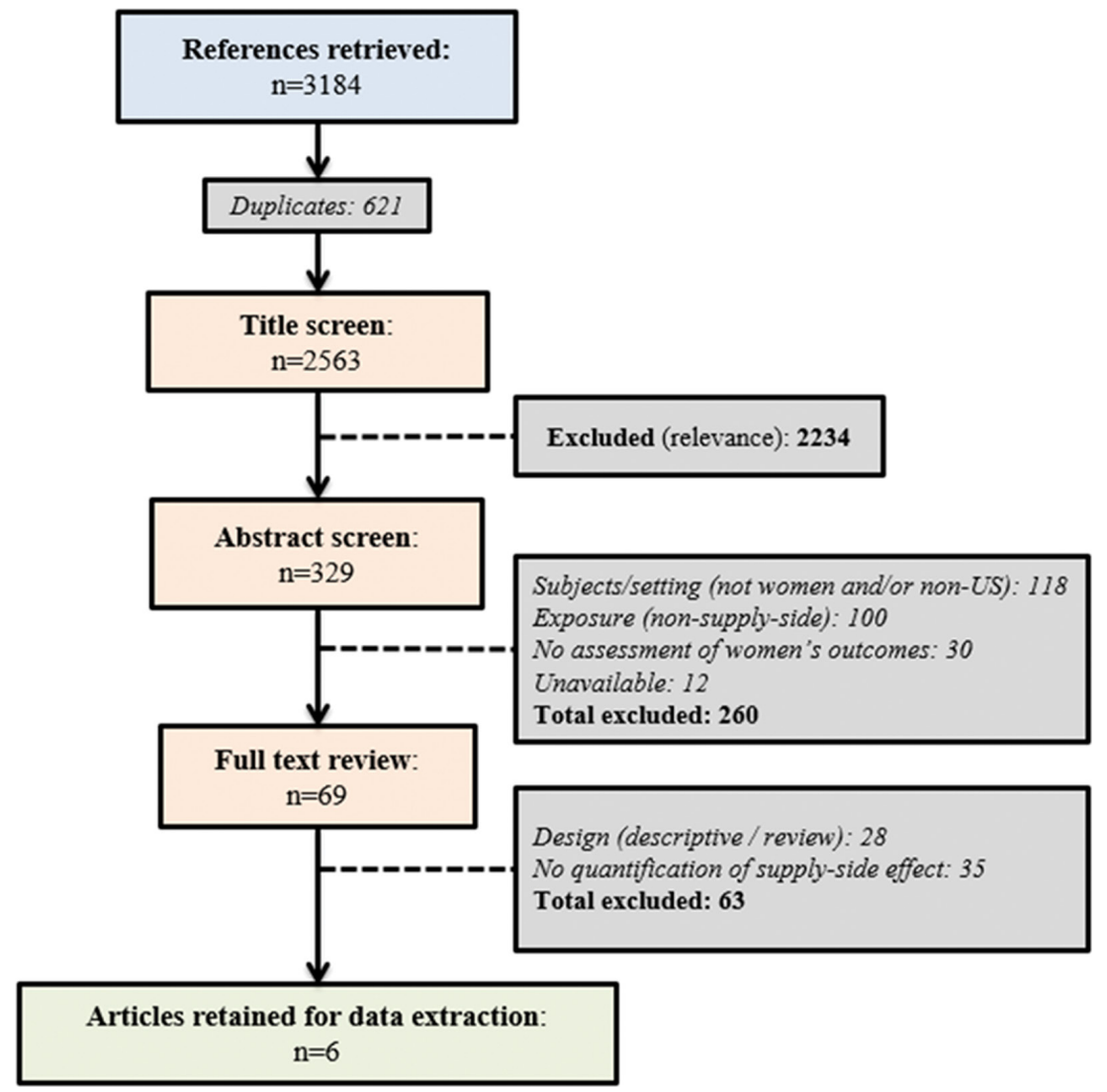

Figure 1 Flow diagram of search process.

included in a full-text review. Just six articles met our inclusion criteria (flow diagram, figure 1); these articles are summarised in table 1 . The mean NOS score was 7.7 (range 6-9), which is generally indicative of moderate to good overall quality. There was considerable heterogeneity across studies, particularly with respect to exposure and outcome definition; this was anticipated given the variety of individual TRAP laws and the wide range of possible health outcomes. Because of these differences, reported findings were not amenable to pooling; any attempt to quantitatively combine these studies would produce vague and potentially misleading effect estimates. We therefore present a narrative synthesis as this is a more appropriate, and we would argue more useful, approach to summarising a relatively diverse collection of literature.

Half of the retained publications featured national-level analyses, and the other half focused specifically on Texas, which was unsurprising given the state's recent and highly-publicised legislative activity. Two of the Texas-based studies ${ }^{517}$ assessed the impact of House Bill 2 (HB2), a group of abortion restrictions enacted in Texas in 2013. HB2's enforced provisions included an admitting privilege requirement, a ban on abortions after 20 weeks' gestation, and restrictions on medication abortion. The third Texas-based analysis ${ }^{18}$ examined the effects of an earlier law - the Women's Right to Know Act (WRTK) - which stipulated that all abortions at or after 16 weeks' gestation must be performed in an
ASC. Studies at the national level assessed a comparatively wider range of regulations: one ${ }^{19}$ used data from NARAL (a reproductive rights advocacy group) to group state-level TRAP laws into two categories (licensing fees, plant/personnel laws), and another ${ }^{20}$ used the same source but grouped TRAP laws according to a six-category classification scheme. The remaining study ${ }^{21}$ assessed the impact of supply-side regulations indirectly by simulating the effect of TRAP-driven provider closures on the national scale.

The most common health outcome was population-level abortion trends (abortion rates/ratios), but there was disagreement among the studies in our sample with respect to the effect of TRAP laws on these outcomes. Three of the five articles that assessed abortion outcomes reported an inverse association between certain TRAP laws and abortion rates, with exposure linked to a decrease in abortion. ${ }^{518} 21$ Depending on the analysis, the magnitude of this effect ranged from a $6.8 \%$ decline in the abortion rate among women aged $18-30$ years, ${ }^{21}$ to a $13 \%$ decline in the abortion rate among all women of childbearing age (15-44 years), ${ }^{5}$ to a $72 \%$ decrease in the abortion rate among women presenting at or after 16 weeks' gestation. ${ }^{18}$ In contrast, one study in this subgroup found no effect of TRAP laws on abortion demand, ${ }^{19}$ and one reported a potential increase in abortions following TRAP legislation. ${ }^{20}$ Both of these studies concluded that demand-side policies (particularly parental consent laws) play a more 


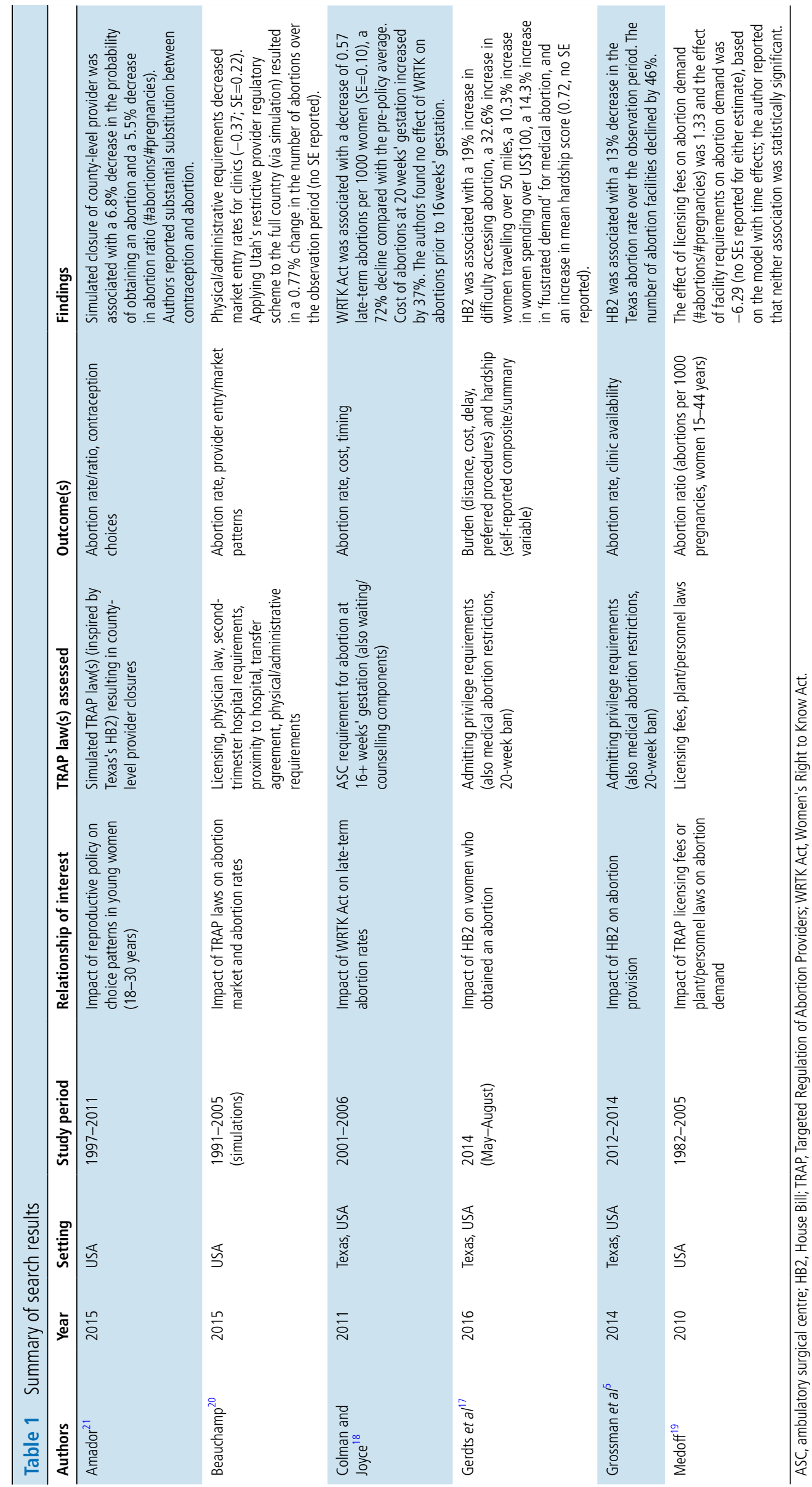


significant role than supply-side policies in explaining the contemporary decline in abortion rates. ${ }^{1920}$

Several studies assessed other relevant outcomes, including gestational age at presentation, out-of-state travel for services, and the cost of, and challenges associated with, obtaining an abortion. Collective findings suggest that aside from any impact TRAP laws may have on abortion rates, these policies may have a meaningful impact on the experience of obtaining an abortion. One study reported a 300\% increase in the number of women travelling out of Texas to obtain abortions at or beyond 16 weeks' gestation in the year following the WRTK Act. ${ }^{18}$ Other publications in our review did not offer a comparable estimate of out-ofstate abortion, but one noted that this phenomenon may become increasingly rare among Texas residents given recently-enacted TRAP polices in neighbouring states. ${ }^{5}$ The clinic closures triggered by HB2 increased within-state travel burdens, ${ }^{517}$ with one study reporting an average four-fold increase in the distance to the closest provider. ${ }^{17}$ HB2 was also linked to increased costs ${ }^{5} 17$ and an increase in women's self-reported hardship in obtaining abortions. ${ }^{17}$ However, while one analysis ${ }^{17}$ found no association between HB2 and gestational age at presentation, the other analysis of the same policy ${ }^{5}$ reported a slight increase in second-trimester abortion.

Although it was not one of our outcomes of interest, most of the articles in this review assessed the impact of TRAP laws on provider availability, which is likely an important mediator in any relationship between TRAP laws and subsequent health outcomes. Studies in our review tended to agree that TRAP laws were associated with a decrease in provider availability, with one reporting a loss of $46 \%$ of all Texas providers in the year following HB2 enforcement. ${ }^{5}$ The relationship between policies and providers was particularly central to one analysis ${ }^{20}$ that was principally concerned with the behaviour of the abortion market in response to regulations. This study reported that TRAP laws decreased market entry rates for clinics and increased the cost of procedures by approximately $10 \%$. The collective findings suggested that a key impact of TRAP laws was not only to close clinics, but also to raise costs for abortion-seeking women because of those closures. ${ }^{171821}$

\section{DISCUSSION}

TRAP laws are widespread in the USA, but quantitative evidence on their effects on women's health remains fairly sparse. The relationship between TRAP laws and abortion outcomes emerged as a central theme in this review: certain TRAP laws - specifically admitting privilege and ASC requirements - were associated with a decrease in abortion rates. However, articles assessing other types (or combinations) of TRAP legislation produced contrary findings. This discordance was probably driven by differences in exposure definition, which suggests that how TRAP exposure is framed has important implications for subsequent conclusions about the laws' effects on abortion outcomes: composite exposure definitions could compromise the ability to quantify the impact of especially deleterious TRAP laws. Given the results of our review, we argue that admitting privilege laws and ASC regulations merit additional research, particularly beyond Texas, as these laws have been enforced in many other states.

We found relatively little evidence on the impact of TRAP laws on other health outcomes. While supporters of these laws argue that extensive regulatory efforts are important in protecting women's health, we did not find any evidence of this impact; however, given the noted safety of abortion in the absence of these policies, ${ }^{4}$ it would be difficult to detect a protective effect if one existed. We did, however, find some limited evidence on the adverse effects of certain laws, many of which are likely to be linked to TRAP-associated increases in the average distance to providers. Our review suggested that certain TRAP laws may be associated with an increase in gestational age at presentation, self-reported hardship, and costs incurred in obtaining abortion, ${ }^{5} 17$ although additional evidence is required to better understand these effects. As abortion becomes more restricted and costly after the first trimester, a substantial increase in gestational age at presentation would be especially troubling. We acknowledge that cost is not a health outcome, but it is almost certainly a key factor on any pathway between policy exposure and abortion or health outcomes. If TRAP laws increase out-of-pocket costs to women, as our collective findings suggest, women of lower socioeconomic position may face greater challenges in obtaining abortions.

We did not identify serious quality concerns over the course of our review, but we did document a number of potential sources of bias and practical issues. Although all of the articles in our review were published in 2010 or later, half ${ }^{18-20}$ assessed policy effects from 2006 or earlier, possibly pre-dating the more recent surge in TRAP enactment. This is likely to be due in large part to delays in abortion data availability and a lack of highquality longitudinal data on state-level TRAP enforcement. Nevertheless, given modern trends in TRAP enforcement and currently available abortion data, it will be useful to re-evaluate existing estimates in the light of contemporary policy shifts. Furthermore, standard errors and confidence intervals were rarely reported, which complicates estimate comparison across studies. There was some disagreement between studies with respect to policy timing, ${ }^{19}{ }^{20}$ which may be due to differences in policy categorisation. It is important to note that narrowing the exposure definition to a single policy or subset of policies may still present challenges, since specific TRAP laws (eg, ASC requirements) vary considerably from state to state. ${ }^{18}$ This complicates interstate analyses or comparisons, and suggests that the findings from the two studies in our review that focused on the admitting privilege requirement of HB2 in Texas may be internally valid, but not generalisable to other states. 
Study designs and target populations also varied, which is an important consideration when interpreting policy effects. For example, findings from the survey-based study of women who successfully obtained abortions ${ }^{17}$ are unlikely to apply to the general population of abortion-seeking women, as women who were unable to obtain abortions were not represented. Likewise, evidence on supply-side policies regulating abortion in the second and third trimester ${ }^{18}$ is probably not generalisable to the broader population, as the majority of US abortions are in the first trimester. ${ }^{7}$ Simulation-based approaches were used in two of the studies in our sample. ${ }^{20} 21$ Although simulation is certainly a valuable tool, given recent policy trends there is an argument for prioritising analyses of observed data. It is also important to note that the effects of TRAP laws may be time-dependent: the extent to which these laws impact abortion rates and other outcomes may depend on the speed (and success) of providers' response to new regulations. ${ }^{18}$ Analyses conducted shortly after a policy shift ${ }^{517}$ may overestimate the long-term impact of a policy if providers are slow to adapt. Finally, TRAP enactment may be precipitated by a shift in public opinion on abortion or a shift in abortion trends; this is an important and generally overlooked potential source of bias, ${ }^{20}$ and should be formally considered in future analyses.

Our review has some limitations. Our search strategy may not have captured all relevant literature on TRAP laws and health; we attempted to mitigate this risk by using multiple search engines and manually searching the reference lists of relevant articles. Our inclusion criteria were also fairly stringent at the full-text stage: there is a broader body of literature on the effects of provider availability on women's outcomes, but these articles generally fell outside our inclusion criteria as clinic closures were not associated with TRAP enactment/enforcement. We also excluded an article on the hypothetical impact of an admitting privilege law in Louisiana, ${ }^{22}$ as the law had not yet taken effect. These exclusions may have omitted relevant information from our narrative synthesis, and they clearly contributed to the low number of articles retained for data extraction, but we believe they were essential in maintaining the integrity of our review. In focusing specifically on TRAP laws, we excluded other supply-side policies from our synthesis, some of which may also have an important impact on abortion trends: for example, one of the studies in our review reported a $70 \%$ decrease in medical abortion following enforcement of HB2, which was likely to have been attributable to policy-driven changes in the availability and cost of mifepristone. ${ }^{5}$ Finally, although geographic and other access-oriented barriers to abortion are not unique to the USA, TRAP laws are a US phenomenon; as such, our findings may not be generalisable to other countries.

\section{CONCLUSIONS}

In 2016, the US Supreme Court determined that the ASC and admitting privilege requirements in Texas' HB2 were unconstitutional, ${ }^{14}$ which effectively opens

\section{Additional educational resources}

Overview of TRAP laws (Guttmacher Institute): https:// www.apha.org/policies-and-advocacy/public-healthpolicy-statements/policy-database/2015/12/14/11/04/ opposition-to-requirements-for-hospital-admittingprivileges-for-abortion-providers

- Opposition to requirements for hospital admitting privileges and transfer agreements for abortion providers (American Public Health Association):https:// www.apha.org/policies-and-advocacy/public-healthpolicy-statements/policy-database/2015/12/14/11/04/ opposition-to-requirements-for-hospital-admittingprivileges-for-abortion-providers

the door to overturning many similar TRAP laws across the country. However, this process does not occur automatically: state-level policy changes will probably take some time to occur, and decreases in provider availability initially driven by TRAP enforcement may persist well into the future. The current political climate in the USA may further delay this process.

Our findings suggest that certain TRAP laws may have an impact on state-level abortion rates, especially in the years immediately following enforcement. These laws may also alter the overall experience of obtaining an abortion. However, additional high-quality research is required to update our knowledge on the impact of TRAP laws on abortion rates and women's health, particularly given post2005 policy trends. We echo calls from previous work ${ }^{18}$ for a research emphasis on specific TRAP laws, such as ASC regulation, that may have a uniquely strong effect on state-level abortion rates. Finally, future work should more explicitly assess the potentially amplified effects of these laws on young, low-income and rural women.

Acknowledgements The authors are grateful to Julia McGarry (JM) and Farhad Udwadia (FU) for their assistance with this study, and to the participants/panellists who provided valuable feedback on this work at the 2017 PAA annual meeting.

Funding NA was supported by a doctoral award from the Fonds de recherche du Québec - Santé (FRQS). SH was supported by at Chercheur Boursier Junior 2 award from the Fonds de recherche du Québec - Santé (FRQS).

Competing interests None declared.

Patient consent Not required.

Provenance and peer review Not commissioned; externally peer reviewed.

(C) Article author(s) (or their employer(s) unless otherwise stated in the text of the article) 2018. All rights reserved. No commercial use is permitted unless otherwise expressly granted.

\section{REFERENCES}

1 Joyce T. The supply-side economics of abortion. N Engl J Med 2011;365:1466-9.

2 Nash E. Policy Trends in the States: 2016. New York: Guttmacher Institute, 2017. https://www.guttmacher.org/ article/2017/01/policy-trends-states-2016. (accessed Jan 2017). 
3 Borgmann CE. Borrowing from dormant commerce clause doctrine in analyzing abortion clinic regulations. Health Matrix Clevel 2016;26:41-64.

4 Charo RA. Whole women's victory - or not? N Engl J Med Overseas Ed 2016;375:809-11.

5 Grossman D, Baum S, Fuentes L, et al. Change in abortion services after implementation of a restrictive law in Texas. Contraception 2014;90:496-501.

6 American College of Obstetricians and Gynecologists. Increasing access to abortion: Committee Opinion No. 613. Obstet Gynecol 2014;124:1060-5.

7 Jones RK, Jerman J. Abortion incidence and service availability in the United States, 2014. Perspect Sex Reprod Health 2017;49:17-27.

8 Joyce T, Tan R, Zhang Y. Abortion before \& after Roe. J Health Econ 2013;32:804-15.

9 Jerman J, Jones RK. Secondary measures of access to abortion services in the United States, 2011 and 2012: gestational age limits, cost, and harassment. Womens Health Issues 2014;24:e419-24.

10 Bearak JM, Burke KL, Jones RK. Disparities and change over time in distance women would need to travel to have an abortion in the USA: a spatial analysis. Lancet Public Health 2017;2:e493-500.

11 Henshaw SK, Joyce TJ, Dennis A, et al. Restrictions on Medicaid funding for abortions: a literature review. New York: Guttmacher Institute, 2009.

12 Joyce TJ, Henshaw SK, Dennis A, et al. The impact of state mandatory counseling and waiting period laws on abortion: a literature review. New York: Guttmacher Institute, 2009.
13 Joyce TJ, Henshaw SK, Dennis A, et al. The impact of laws requiring parental involvement for abortion: a literature review. New York: Guttmacher Institute, 2009.

14 Whole Woman's Health v Hellerstedt, 579 US. 2016.

15 Wells GA, Shea B, O'Connell D, et al; The Newcastle-Ottawa Scale (NOS) for assessing the quality of nonrandomised studies in meta-analyses. Ottawa, ON: Ottawa Hospital Research Institute, 2011. http:/www.ohri.ca/programs/clinical_ epidemiology/oxford.asp (accessed Oct 2017).

16 Bae JM. A suggestion for quality assessment in systematic reviews of observational studies in nutritional epidemiology. Epidemiol Health 2016;38:e2016014-6.

17 Gerdts C, Fuentes L, Grossman D, et al. Impact of clinic closures on women obtaining abortion services after implementation of a restrictive law in Texas. Am J Public Health 2016;106:857-64.

18 Colman S, Joyce T. Regulating abortion: impact on patients and providers in Texas. J Policy Anal Manag 2011;30:775-97.

19 Medoff MH. State abortion policies, targeted regulation of abortion provider laws, and abortion demand. Rev Policy Res 2010;27:577-94.

20 Beauchamp A. Regulation, imperfect competition, and the US abortion market.. Int Econ Rev 2015;56:963-96.

21 Amador D. The consequences of abortion and contraception policies on young women's reproductive choices, schooling and labor supply. Publicly Accessible Penn Dissertations. 2015.

22 Roberts SC, Fuentes L, Kriz R, et al. Implications for women of Louisiana's law requiring abortion providers to have hospital admitting privileges. Contraception 2015;91:368-72.

\section{Commentary}

For numbered affiliations, see end of the article.

\section{Correspondence to}

Dr Ganesh Acharya, Department of Clinical Science, Intervention and Technology (CLINTEC), Karolinska Institute and Centre for Fetal Medicine, Karolinska University Hospital, Stockholm SE-141 86, Sweden; ganesh. acharya@ki.se

Received 1 February 2018 Revised 5 February 2018 Accepted 15 February 2018

\section{Linked}

- http://dx.doi.org/10.1136/ bmjsrh-2017-101866

Check for updates

To cite: Acharya G, Liang $\mathrm{H}$. BMJ Sex Reprod Health 2018;44:134-135.

\section{Abortion politics: do TRAP laws have an impact on women's health?}

\author{
Ganesh Acharya, ${ }^{1}$ Huan Liang ${ }^{1,2}$
}

Abortion is regulated by law in most (if not all) countries, with a view to preventing abortion except under defined circumstances, and ensuring that abortions are performed safely. Abortion is allowed in 97\% of the United Nations' member states in order to save pregnant women's lives. ${ }^{1}$ Today abortions are increasingly provided by healthcare personnel other than doctors, in community settings or even at home rather than in hospitals, ${ }^{23}$ and have become very safe in most countries where they are legal and accessible. ${ }^{24}$ However, regulations vary significantly around the world. ${ }^{1}$

In considering what legal position should be advocated and how regulation affects women's health, it should be remembered that provision of safe abortion services was driven by the public health necessity to reduce maternal mortality, not as a primary human rights issue. The proportion of unsafe abortions is significantly higher in countries with highly restrictive abortion laws compared with those with liberal and less restrictive laws. ${ }^{4}$ Maternal mortality still remains unacceptably high in countries where abortion is illegal.

Even in some highly developed, democratic and affluent countries, such as the UK and the USA, complete decriminalisation of abortion has not been achieved yet, and policies that impose impractical, 
costly and resource-intensive requirements on abortion providers may have a negative impact on abortion accessibility and on women's health. However, reducing abortion numbers is also a worthwhile goal from the perspective of health policymakers, and of women themselves. How best to achieve that continues to dominate the political debate, especially in the USA.

Governments' priorities and policies have an impact on both health and healthcare ethics. Undoubtedly, educating girls and women and providing easy access to birth control is an effective way of reducing the need for an abortion. However, enforcing policies such as so called Targeted Regulation of Abortion Providers (TRAP) laws may have a negative impact on the accessibility of abortion services, and is unlikely to support women's health. Conversely, a government policy that encourages women to have an abortion to meet national family planning targets or a society that expects women to abort a female fetus cannot be considered humane or ethical even if the access to abortion services is excellent and procedures are medically safe.

The systematic review by Austin and Harper ${ }^{5}$ published in this issue of the journal aimed to evaluate the impact of TRAP laws on population-level abortion trends, gestational age at presentation, and measures of self-perceived burden. The authors conclude that certain TRAP laws may have an impact on state-level abortion rates and the experience of obtaining an abortion in the USA.

The study is limited by small numbers $(n=6)$ of included studies, and heterogeneity in design, methodology and reporting, preventing quantitative measurement of the impact of exposure to TRAP laws (composite or individual components) and meta-analysis. The resulting narrative synthesis inevitably carries the risk of desirability bias and limited generalisability. This systematic review does provide a welcome reminder that TRAP laws are not solely a US-specific phenomenon and their impact on abortion services needs to be investigated in other settings too. However, given abortion is already known to be safe in deregulated settings, the prior probability that extensive regulatory efforts will benefit women's health is very low. It may, rather, be a question of measuring the degree of detriment.

\section{Author affiliations \\ ${ }^{1}$ Division of Obstetrics and Gynecology, Department of Clinical Science Intervention and Technology, Karolinska Institutet and Center for Fetal Medicine, Karolinska University Hospital, Stockhom, Sweden \\ ${ }^{2}$ Department of Obstetrics, Obstetrics and Gynecology Hospital of Fudan University, Shanghai, China}

Funding This research received no specific grant from any funding agency in the public, commercial or not-for-profit sectors.

Competing interests None declared.

Patient consent Not required.

Provenance and peer review Commissioned; internally peer reviewed.

(C) Article author(s) (or their employer(s) unless otherwise stated in the text of the article) 2018. All rights reserved. No commercial use is permitted unless otherwise expressly granted.

\section{REFERENCES}

1 United Nations, Department of Economic and Social Affairs, Population Division. Abortion policies and reproductive health around the world. United Nations publication, 2014. Sales No. E.14.XIII.11. http://www.un.org/en/development/desa/populatio $\mathrm{n} /$ publications/pdf/policy/AbortionPoliciesReproductiveHealth. pdf (accessed 5 Feb 2018).

2 Barnard S, Kim C, Park MH, et al. Doctors or mid-level providers for abortion. Cochrane Database Syst Rev 2015;7:CD011242.

3 Rocca CH, Puri M, Shrestha P, et al. Effectiveness and safety of early medication abortion provided in pharmacies by auxiliary nurse-midwives: a non-inferiority study in Nepal. PLoS One 2018;13:e0191174.

4 Ganatra B, Gerdts C, Rossier C, et al. Global, regional, and subregional classification of abortions by safety, 201014: estimates from a Bayesian hierarchical model. Lancet 2017;390:2372-81.

5 Austin N, Harper S. Assessing the impact of TRAP laws on abortion and women's health in the USA: a systematic review. BMJ Sex Reprod Health 2018;44:129-34.

\section{Informative or amusing fillers invited}

Sometimes, in the process of finalising each journal issue for print publication, we are left with blank spaces at the end of artides - like this one. We like to make good use of these spaces when we can, and thus welcome 'fillers' of up to 250 words which inform or entertain.

These fillers can be factual, funny, challenging or creative, but they need to relate to sexual health. Have you come across something wise, informative or amusing on social media which is relavant to our readers? Have you learned something in another field that you think SRH practitioners might benefit from? Have you heard something throughtprovoking in conversation, or have you a haiku up your sleeve?

We cannot guarantee publication, but welcome all ideas and submissions, and will publish these where suitable, and as space in a print journal issue allows. We will let you know if we are unable to publish your contribution for any reason.

All submissions should be submitted to the Journal Editorial Office at Journal@fsrh.org 